Appendix of our article, available at NEJM.org). In addition to treatment group, histologic findings emerged as an independent variable with regard to both progression-free survival and overall survival. Extent of resection dropped out during the stepwise selection process for both. Consequently, it is unlikely that either variable confounded the results.

Since radiation therapy can cause neurocognitive decline, the appropriate time to initiate chemotherapy plus radiotherapy or radiotherapy remains controversial. Patients in the RTOG 9802 trial were randomly assigned to begin radiotherapy alone or chemotherapy plus radiotherapy within 4 weeks after surgery. The trial was not designed to address the question of timing of radiotherapy.

Neurocognitive end points from the trial were reported previously and showed no worsening of mean Mini-Mental State Examination scores in either treatment group up to 5 years after the initiation of treatment. ${ }^{1}$ These data do not address less severe or more delayed neurocognitive decline.

In the randomized groups, $48 \%$ of the patients underwent biopsy only. Low-grade gliomas are characterized by low cellularity, which results in small amounts of available tissue. The two studies ${ }^{2,3}$ cited by Touat et al. enrolled patients with tumors with anaplastic oligodendroglial elements rather than those with lower-grade oligodendroglial or astrocytic tumors (as were enrolled in the RTOG 9802 trial). In addition, when the trial began 18 years ago, institutional processes for collecting and submitting tumor tissue were less well developed than they are today. As a consequence, we had insufficient data for this report to address the effect of chemotherapy plus radiotherapy in patients with IDH1/2 wild-type low-grade gliomas. Efforts are under way to obtain tissues to increase the pre- dictive power of future correlative analyses from the RTOG 9802 trial.

We remind Knisely and Schulder that $63 \%$ of the patients with tumors that were tested had tumoral IDH1 R132H mutations. In this subgroup, survival was longer among patients who received chemotherapy plus radiotherapy than among those who received radiotherapy alone (hazard ratio, 0.42; $\mathrm{P}=0.02$ ). There is clear evidence to support treatment recommendations on the basis of current molecular classification for the majority of patients with low-grade glioma. Given the results of the RTOG 9402 and EORTC 26951 trials, ${ }^{2,3}$ it is reasonable to expect a benefit with PCV in patients with either low-grade or anaplastic gliomas containing other tumoral IDH1/2 mutations and little reason to expect a benefit in those with IDH1/2 wild-type tumors.

Jan C. Buckner, M.D.

Mayo Clinic

Rochester, MN

buckner.jan@mayo.edu

Arnab Chakravarti, M.D.

Ohio State University

Columbus, $\mathrm{OH}$

Walter J. Curran, Jr., M.D.

Winship Cancer Institute of Emory University

Atlanta, GA

Since publication of their article, the authors report no further potential conflict of interest.

1. Prabhu RS, Won M, Shaw EG, et al. Effect of the addition of chemotherapy to radiotherapy on cognitive function in patients with low-grade glioma: secondary analysis of RTOG 98-02. J Clin Oncol 2014;32:535-41.

2. Cairncross JG, Wang M, Jenkins RB, et al. Benefit from procarbazine, lomustine, and vincristine in oligodendroglial tumors is associated with mutation of IDH. J Clin Oncol 2014;32:783-90. 3. van den Bent MJ, Brandes AA, Taphoorn MJ, et al. Adjuvant procarbazine, lomustine, and vincristine chemotherapy in newly diagnosed anaplastic oligodendroglioma: long-term follow-up of EORTC brain tumor group study 26951. J Clin Oncol 2013;31:34450

DOI: $10.1056 / N E J M c 1605897$

\title{
Management of Advanced Head and Neck Cancer
}

TO THE EDITOR: The PET-NECK randomized trial by Mehanna et al. (April 14 issue) ${ }^{1}$ compared surveillance guided by positron-emission tomography-computed tomography (PET-CT) with planned neck dissection after chemoradiotherapy in patients with head and neck squamous-cell carcinoma (HNSCC) and stage N2 or N3 nodal metas- tases. Patients who had a complete metabolic response but residual lymphadenopathy underwent neck dissection. A complete metabolic response on PET-CT in the presence of residual enlarged nodes has a high negative predictive value in oropharyngeal carcinoma associated with human papillomavirus (HPV). ${ }^{2}$ Therefore, although these 
patients would have undergone neck dissection according to the trial protocol, Mehanna et al. suggest considering imaging follow-up in this patient subgroup.

A critical question is whether this approach can be extrapolated for non-HPV-related HNSCC with a complete metabolic response and residual adenopathy on PET-CT. Other studies have shown a high negative predictive value for a complete metabolic response on PET-CT in patients with residual nodal tissue and non-HPV-related cancers. ${ }^{3,4}$ Scoring systems for metabolic response assessment that are similar to those routinely used in patients with lymphoma have been devised. ${ }^{4}$ Data regarding the safety and cost-effectiveness of less intensive clinical follow-up in patients with HNSCC who have negative results on PET-CT after treatment have been reported. ${ }^{5}$ Determining whether metabolically inactive residual lymph-node tissue can be safely observed for various HNSCC sites is an important priority.

Robin J.D. Prestwich, Ph.D., M.D.

Mehmet Sen, M.D.

Andrew F. Scarsbrook, M.D.

Leeds Cancer Centre

Leeds, United Kingdom

robin.prestwich@nhs.net

No potential conflict of interest relevant to this letter was reported.

1. Mehanna H, Wong W-L, McConkey CC, et al. PET-CT surveillance versus neck dissection in advanced head and neck cancer. N Engl J Med 2016;374:1444-54.

2. Vainshtein JM, Spector ME, Stenmark MH, et al. Reliability of post-chemoradiotherapy F-18-FDG PET/CT for prediction of locoregional failure in human papillomavirus-associated oropharyngeal cancer. Oral Oncol 2014;50:234-9.

3. Slevin F, Subesinghe M, Ramasamy S, Sen M, Scarsbrook AF, Prestwich RJ. Assessment of outcomes with delayed (18)F-FDG PET-CT response assessment in head and neck squamous cell carcinoma. Br J Radiol 2015;88:20140592.

4. Marcus C, Ciarallo A, Tahari AK, et al. Head and neck PET/ CT: therapy response interpretation criteria (Hopkins Criteria) — interreader reliability, accuracy, and survival outcomes. J Nucl Med 2014;55:1411-6.

5. Shah K, Te Marvelde L, Collins M, et al. Safety and cost analysis of an (18)FDG-PET-CT response based follow-up strategy for head and neck cancers treated with primary radiation or chemoradiation. Oral Oncol 2015;51:529-35.

DOI: 10.1056/NEJMc1606211

TO THE EDITOR: The PET-NECK trial by Mehanna et al. provides the first randomized trial evidence to support the routine use of PET-CT in lieu of planned neck dissection after a favorable response to definitive chemoradiation in patients with locoregionally advanced head and neck cancer. This trial builds on previous retrospective and obser- vational data to show that surveillance PET-CT is associated with rates of locoregional control and overall survival that are similar to those associated with planned neck dissection for patients with stage $\mathrm{N} 2$ or $\mathrm{N} 3$ nodal metastases who have resolution of neck disease after chemoradiation. Unfortunately, the authors do not report the rates of residual pathologically positive lymph nodes at the time of neck dissection in either study group, nor do they report these rates stratified according to tumor p16 status. This information, as well as factors associated with positive residual disease, would be important for clinicians in the interpretation of both the study results and equivocal findings on surveillance PET-CT in practice. The authors provide recommendations for treating patients with such equivocal findings on the basis of tumor p16 status, but these recommendations could be fortified with data from the current study.

Caleb Dulaney, M.D.

Lauren Kropp, M.D.

James Bonner, M.D.

University of Alabama at Birmingham

Birmingham, AL

jabonner@uabmc.edu

No potential conflict of interest relevant to this letter was reported.

DOI: 10.1056/NEJMc1606211

the authors Reply: We agree with Prestwich et al. that the question of whether metabolically inactive residual lymph-node tissue can be safely observed in various HNSCC sites is an important one. Currently available data for both HPV-positive and HPV-negative disease have been derived mainly from retrospective, relatively small, nonrandomized studies. When new data become available, the findings may spare a small number of additional patients from undergoing neck dissection.

We respectfully disagree with Dulaney et al. regarding the need for histopathological results on lymph-node status. It is widely accepted that there is no reliable way of identifying whether residual nodal tumor that is identified on histologic analysis after chemoradiotherapy represents viable disease. ${ }^{1}$ Therefore, the use of such analysis as an end point leads to an overestimation of positivity rates and has been one of the main weaknesses in the studies that have been published to date. In our study, we sought to address this weakness by the use of clinically relevant primary and secondary outcomes — rates of over- 
all and disease-free survival. Finally, we reported data in our study for both HPV-positive and HPV-negative disease that showed the efficacy of active surveillance for both types of tumors.

Hisham Mehanna, Ph.D.

University of Birmingham

Birmingham, United Kingdom

h.mehanna@bham.ac.uk

Wai-Lup Wong, F.R.C.R.

Mount Vernon Hospital

Northwood, United Kingdom
Janet Dunn, Ph.D.

University of Warwick

Coventry, United Kingdom

Since publication of their article, the authors report no further potential conflict of interest.

1. Porceddu SV, Pryor DI, Burmeister E, et al. Results of a prospective study of positron emission tomography-directed management of residual nodal abnormalities in node-positive head and neck cancer after definitive radiotherapy with or without systemic therapy. Head Neck 2011;33:1675-82.

DOI: 10.1056/NEJMc1606211

\section{The Hospital Readmissions Reduction Program}

TO THE EDITOR: Zuckerman et al. (April 21 issue) ${ }^{1}$ found that hospital readmissions for conditions targeted for penalties fell by 3.7 percentage points, whereas stays in observation units rose by 2.1 percentage points, yet they concluded that these two trends were not related because observation stays were rising even before the penalties were in place. Instead, the authors attribute continuously rising observation rates to hospitals' confusion over the criteria used in audits of inpatient stays.

It seems unlikely that confusion over these regulations continued to increase for many years, leading hospitals to sacrifice billions by billing for observation stays rather than for more lucrative admissions. More likely, hospital executives realized that, on balance, avoidance of readmission penalties by relabeling inpatient stays as "observation" was the most lucrative strategy.

The authors also overinterpret their statistics as showing no correlation between readmissions and observation stays. In fact, they, like previous analysts, ${ }^{2}$ found a weak positive correlation $(\mathrm{P}=0.07)$ - indicating a $93 \%$ likelihood that falling readmissions and rising observations were related. Finally, their analysis ignores other potential gaming strategies - for example, upcoding coexisting conditions to improve riskadjusted rates and shifting inpatient-type care to emergency departments.

\section{Steffie Woolhandler, M.D., M.P.H.}

\section{David U. Himmelstein, M.D.}

City University of New York School of Public Health at Hunter College New York, NY

dhimmels@hunter.cuny.edu

No potential conflict of interest relevant to this letter was reported.

1. Zuckerman RB, Sheingold SH, Orav EJ, Ruhter J, Epstein AM.
Readmissions, observation, and the Hospital Readmissions Reduction Program. N Engl J Med 2016;374:1543-51.

2. Yale New Haven Health Services Corporation Center for Outcomes Research and Evaluation. Medicare hospital quality chartbook: performance report on outcome measures. Baltimore: Centers for Medicare \& Medicaid Services, September 2014 (https:/ www.cms.gov/Medicare/Quality-Initiatives-Patient-Assessment -Instruments/HospitalQualityInits/Downloads/Medicare-Hospital -Quality-Chartbook-2014.pdf).

DOI: 10.1056/NEJMc1606658

TO THE EDITOR: Zuckerman et al. examined changes in readmission rates and the use of observation status after the 2012 initiation of financial penalties under the Medicare Hospital Readmissions Reduction Program (HRRP). Their analysis was stratified according to HRRP targeted conditions (acute myocardial infarction, heart failure, and pneumonia) versus all others. Gaining a better understanding of readmissions is useful for a variety of Centers for Medicare and Medicaid Services programs, including the HRRP, the Hospital Value-Based Purchasing Program, and the Bundled Payments for Care Improvement (BPCI) Initiative. Although some policymakers seek to expand readmission penalties to all hospitalizations, there is evidence to suggest that certain conditions might be prioritized over others. ${ }^{1}$ It may make sense to start with conditions covered by the BPCI initiative because the program currently has the largest number of conditions treated by a variety of medical and surgical specialties. ${ }^{2}$ Moreover, considerable effort was already undertaken to choose clinically meaningful and policy-relevant conditions for the BCPI program. Information on changes in readmission and observation rates (and the interaction between measures) for these conditions may provide valuable insight to both clinicians and policymakers. 